\title{
1. An ultimate storage ring lattice with vertical emittance generated by damping wigglers
}

Xiaobiao Huang

\section{Abstract}

We discuss the approach of generating round beams for ultimate storage rings using vertical damping wigglers (with horizontal magnetic field). The vertical damping wigglers provide damping and excite vertical emittance. This eliminates the need to generate large linear coupling that is impractical with traditional off-axis injection. We use a PEP-X compatible lattice to demonstrate the approach. This lattice uses separate quadrupole and sextupole magnets with realistic gradient strengths. Intrabeam scattering effects are calculated. The horizontal and vertical emittances are $22.3 \mathrm{pm}$ and $10.3 \mathrm{pm}$, respectively, for a $200 \mathrm{~mA}, 4.5 \mathrm{GeV}$ beam, with a vertical damping wiggler of a total length of 90 meters, peak field of $1.5 \mathrm{~T}$ and wiggler period of $100 \mathrm{~mm}$.

6 Keywords: ultimate storage ring, vertical emittance, damping wiggler

\section{Introduction}

In present day third generation light sources, the vertical emittance is usually small compared to the horizontal emittance. It is typically a few percent of the latter or below without coupling correction and can reach pico-meter level with coupling correction. For ultimate storage rings (USR), it is not advisable to maintain the same level vertical-to-horizontal emittance ratio. This is because the horizontal emittance will already be diffraction-limited and hence there is no need to make the vertical emittance any smaller. In addition, a smaller vertical emittance would cause significant emittance growth due to intrabeam scattering (IBS) and also short Touschek lifetime. Many USR designs to-date








Figure 1: Injection efficiency vs. coupling ratio at SPEAR3.

35

36

(such as PEP-X [3]) assume the vertical emittance to be equal to the horizontal emittance, resulting in a round beam.

Vertical emittance in a storage ring can be generated with linear coupling or vertical dispersion. A round beam can be achieved with 100\% linear coupling, in which case the horizontal and vertical emittances are $50 \%$ of the natural emittance. The reduction of horizontal emittance by a factor of 2 is a significant benefit of this approach. However, large coupling between the two transverse directions will cause injection difficulties for off-axis injection. The injected beam, initially at a large horizontal offset, will take large vertical oscillation and likely get lost to small vertical apertures such as the small-gap insertion devices. Effectively, large coupling with small vertical apertures causes the dynamic aperture to decrease. This is experimentally demonstrated on the SPEAR3 storage ring as is shown in Figure 1, which shows that the injection efficiency drops to zero at or before the coupling ratio is increased to $26 \%$. Large coupling may also reduce the local momentum aperture and in turn the Touschek lifetime for a beam with a given vertical emittance since the horizontal oscillation of the Touschek particles will be coupled to the vertical plane which usually has smaller apertures.

The second approach to generate vertical emittance is to create vertical dispersion inside dipole magnets. This will not cause injection and lifetime 
difficulties, but will lose the benefit of horizontal emittance reduction.

We have studied a third approach which can mitigate the negative effects of both of the above approaches. In this approach we use vertical damping wigglers (with horizontal magnetic field) to achieve both the reduction of horizontal emittance and the generation of vertical emittance. Damping wigglers may be desirable for USRs because the dipole bending radius of USRs could be large and hence the radiation energy loss from dipole magnets may be too small for fast enough damping, which is required for controlling collective effects such as intrabeam scattering and beam instabilities. An additional benefit of damping wigglers is that they can reduce the sensitivity of beam parameters (such as emittances) to status of user insertion devices as they provide a large anchor term to the radiation damping. Usually damping wigglers have vertical magnet field that causes wiggling beam motion on the horizontal plane. The intrinsic horizontal dispersion generated by the damping wiggler itself contributes to an increase of the horizontal emittance (even though overall the emittance may be still decreasing due to extra damping). The relative emittance contribution from the damping wiggler can be significant when the natural emittance is small. Choosing to use small period damping wigglers alleviates the emittance growth problem to some extent. But it puts a challenge to the damping wiggler design and increases the cost. A vertical damping wiggler does not increase the horizontal emittance and in the same time generates the desirable vertical emittance. Therefore it is reasonable to use vertical damping wigglers for USRs. The idea of using vertical damping wigglers to generate vertical emittance has been independently proposed in Refs. [1, 2].

In this study we demonstrate this approach with a lattice that is compatible with the PEP tunnel at SLAC National Accelerator Laboratory. In section 2 we use a simple model to calculate and compare the emittances with the horizontal and vertical damping wiggler approaches. In section 3 the PEP compatible lattice with vertical damping wigglers is presented. Emittance parameters with intrabeam scattering effects are given in section 4. The conclusions are given in section 5 . 


\section{Theoretic calculation}

76 generated by the DW itself is

$$
D_{y}=-\frac{1}{\rho_{w} k^{2}}(1-\cos k s), \quad D_{y}^{\prime}=-\frac{1}{\rho_{w} k} \sin k s,
$$

77 Consequently the radiation integral [5] contributions are

$$
\begin{aligned}
I_{2 w} & =\frac{L_{w}}{2 \rho_{w}^{2}}, \quad I_{3 w}=\frac{4 L_{w}}{3 \pi \rho_{w}^{3}}, \\
I_{4 w y} & =\frac{3 L_{w}}{8 \pi \rho_{w}^{4} k^{2}}, \quad I_{5 w y}=\frac{4<\beta_{y}>L_{w}}{15 \pi \rho_{w}^{5} k^{2}},
\end{aligned}
$$

78 where $\left\langle\beta_{y}\right\rangle$ is the average vertical beta function across the DW. Assuming 79 no lattice errors, the emittances and momentum spread are given by

$$
\begin{aligned}
\sigma_{\delta}^{2} & =\gamma^{2} C_{q} \frac{I_{3}+I_{3 w}}{I_{2}+I_{2 w}} \frac{1}{2+\mathcal{D}_{x}+\mathcal{D}_{y}}, \\
\epsilon_{x} & =\gamma^{2} C_{q} \frac{I_{5}}{I_{2}+I_{2 w}} \frac{1}{1-\mathcal{D}_{x}}, \\
\epsilon_{y} & =\gamma^{2} C_{q} \frac{I_{5 w y}}{I_{2}+I_{2 w}} \frac{1}{1-\mathcal{D}_{y}},
\end{aligned}
$$

80

where $I_{2-5}$ are radiation integrals for the bare lattice and

$$
\mathcal{D}_{x}=\frac{I_{4}}{I_{2}+I_{2 w}}, \quad \mathcal{D}_{y}=\frac{I_{4 w y}}{I_{2}+I_{2 w}} .
$$


We now consider a PEP-X compatible lattice at $4.5 \mathrm{GeV}$ (see section 3). The relevant radiation integrals without DWs are

$$
\begin{aligned}
& I_{2}=0.1026 \mathrm{~m}^{-1}, \quad I_{3}=1.674 \times 10^{-3} \mathrm{~m}^{-2}, \\
& I_{4}=-0.1215 \mathrm{~m}^{-1}, \quad I_{5}=3.092 \times 10^{-7} \mathrm{~m}^{-1} .
\end{aligned}
$$

Assuming the average beta function over the DW is $10 \mathrm{~m}$, the emittances as a function of wiggler length is calculated and compared to the case with a regular horizontal damping wiggler for various sets of peak magnetic field and wiggler period values. The results are shown in Figure 2. Clearly the vertical DW provides damping of the horizontal emittance and in the meantime generates vertical emittance. The total emittance is only slightly larger than the case with a regular horizontal DW. The difference is smaller for smaller wiggler periods.

\section{Application to a PEP-X compatible lattice}

We have applied the vertical DW approach in a design study for a PEP-X compatible lattice with the design beam energy at $4.5 \mathrm{GeV}$. This lattice is similar to the PEP-X USR design as it adopts the same MBA and fourth order achromat approach [3]. The 2.2-km long PEP tunnel has a hexagonal geometry. There are six 120-m long straight sections which can be used to host long damping wigglers. The lattice has 6 arcs, each consists of $8 \mathrm{MBA}$ (with $M=7$ ) cells. An MBA cell is composed of 5 identical TME cells in the middle and two matching cells at the ends. The MBA cell and the TME cell are shown in Figure 3. The TME and 7BA cell lengths are $3.12 \mathrm{~m}$ and $30.4 \mathrm{~m}$, respectively. The TME dipole magnet is $1.12 \mathrm{~m}$ in length and its bending angle is $1.0475^{\circ}$. This dipole is a combinedfunction magnet with a defocusing quadrupole component and the normalized gradient is $-0.7989 \mathrm{~m}^{-2}$. The focusing quadrupole (QF) is split into two halves to put the SF sextupole in between. The length of each half is $0.18 \mathrm{~m}$. The length of SF is $0.30 \mathrm{~m}$. One SD sextupole magnet is put at each end of the dipole. Its length is $0.21 \mathrm{~m}$. The matching dipole has no quadrupole gradient. Its length is $8 \%$ longer than the TME dipole. At each end of the MBA cell, outside of the 

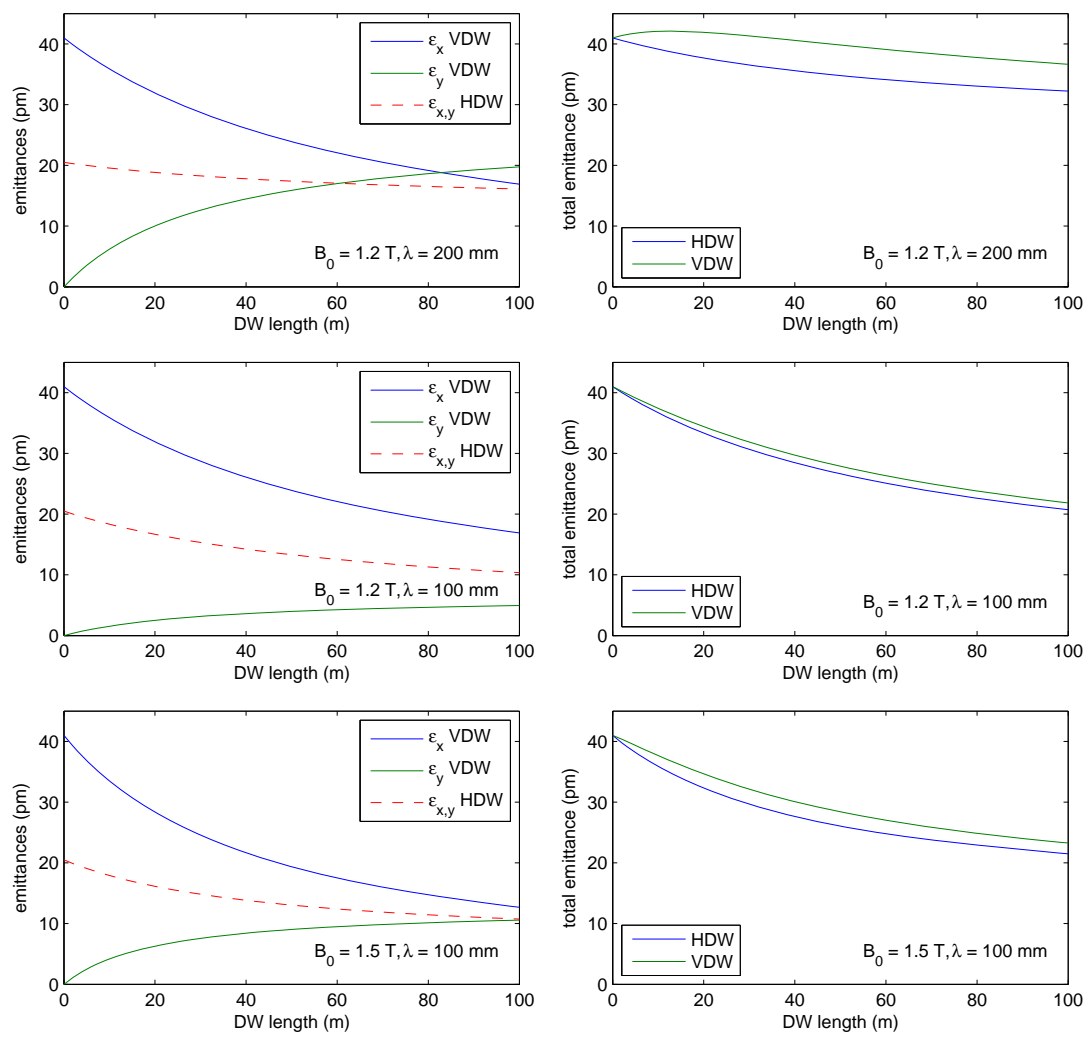

Figure 2: Comparison of the emittances of a PEP-X ring with vertical or horizontal damping wigglers. Top row with $B_{w}=1.2 \mathrm{~T}$ and $\lambda_{w}=200 \mathrm{~mm}$; middle row with $B_{w}=1.2 \mathrm{~T}$ and $\lambda_{w}=100 \mathrm{~mm}$; bottom tow with $B_{w}=1.5 \mathrm{~T}$ and $\lambda_{w}=100 \mathrm{~mm}$. A $100 \%$ coupling (i.e., $\epsilon_{x}=\epsilon_{y}=\epsilon_{x 0} / 2$, where $\epsilon_{x 0}$ is uncoupled horizontal emittance) is assumed for the regular horizontal DW case. 
matching dipole, there is a quadrupole triplet. Three harmonic sextupoles are put between these magnets. The minimum edge-to-edge distance for magnets is $8 \mathrm{~cm}$ to accommodate coils and BPMs [6]. The quadrupole strength is below $51 \mathrm{~T} / \mathrm{m}$ and the sextupole strength is below $7500 \mathrm{~T} / \mathrm{m}^{2}$. With a bore radius of $12.5 \mathrm{~mm}$, the pole tip magnetic field would be below $0.64 \mathrm{~T}$ for quadrupoles and below $0.59 \mathrm{~T}$ for sextupoles.


Figure 3: The TME cell (top) and 7BA cell (bottom) for the PEP-X compatible lattice.

112

113

114

115

The insertion device straight sections between the MBA cells are 5 meter long. The horizontal and vertical beta functions at the centers of these straight sections are $0.8 \mathrm{~m}$ and $2.0 \mathrm{~m}$, respectively. The horizontal beta function is made 

long straight section is as shown in Figure 5.

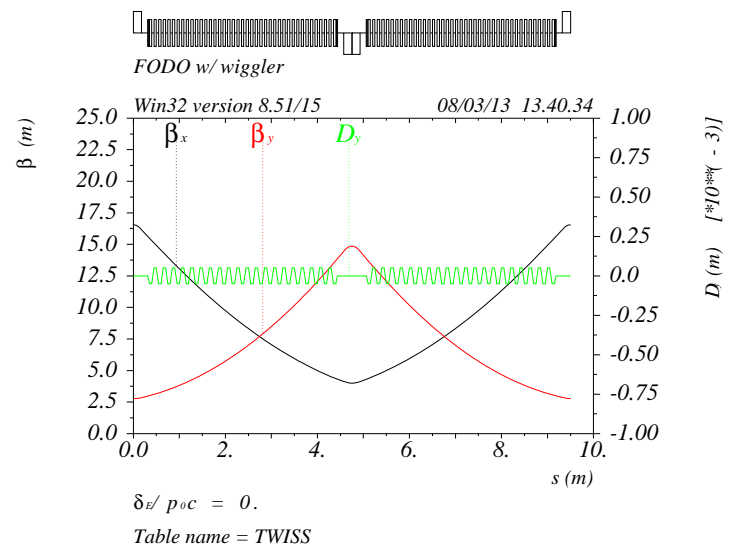

Figure 4: One FODO lattice period with damping wigglers.

124

very small (at the optimal value of $L / 2 \pi=0.8 \mathrm{~m}$ ) to provide better matching of the electron and photon optics. But we keep the vertical beta at a level close to half of the ID straight length to allow small gap insertion devices [7].

The 120-m long straight sections are filled with FODO cells. One of the long straight sections houses the damping wigglers. The wiggler sections are 4.06 meter long and are put between the quadrupoles of the FODO cells. The optics functions are shown in Figure 4 for a FODO cell for the case with wiggler period at $200 \mathrm{~mm}$ and peak field at $1.2 \mathrm{~T}$. Optics function for one half of the

Table name $=$ TWISS

The ring lattice parameters for three wiggler settings are compared in Table 1. The parameters were calculated with MAD8 [8]. The results agree with the analytic prediction given in Figure 2. For the vertical DW sets of $(1.2 \mathrm{~T}$, $200 \mathrm{~mm}$ ) and (1.5 T, $100 \mathrm{~mm})$, the horizontal and vertical emittances are nearly equal, with values down to $17 / 17 \mathrm{pm}$ and $13 / 10 \mathrm{pm}$, respectively.

\section{Intrabeam scattering calculation}

Intrabeam scattering (IBS) can significantly increase the emittance and energy spread for very low emittance beams at high current. To examine how the 


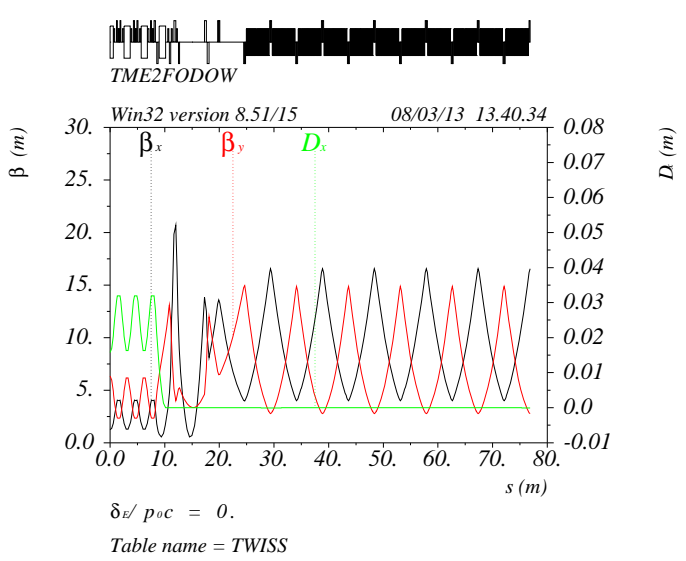

Figure 5: Half of the DW straight section.

Table 1: Ring parameters with or without vertical damping wigglers

\begin{tabular}{l|cccc}
\hline parameters & no DW & VDW-1 & VDW-2 & VDW-3 \\
\hline Energy $(\mathrm{GeV})$ & \multicolumn{4}{|c}{4.5} \\
Circumference $(\mathrm{m})$ & \multicolumn{4}{|c}{2199.3} \\
$\nu_{x, y}$ & \multicolumn{4}{|c}{$0.38 \times 10^{-4}$} \\
$\alpha_{c}$ & \multicolumn{4}{|c}{89.73 .30} \\
\hline VDW length $(\mathrm{m})$ & 0 & 90.8 & 89.4 \\
VDW $B_{w}(\mathrm{~T})$ & & 1.2 & 1.2 & 1.5 \\
VDW $\lambda_{w}(\mathrm{~m})$ & & 0.2 & 0.1 & 0.1 \\
\hline$U_{0}(\mathrm{MeV})$ & 0.59 & 2.30 & 2.26 & 3.19 \\
$\epsilon_{x}(\mathrm{pm})$ & 36.5 & 16.5 & 16.7 & 12.7 \\
$\epsilon_{y}(\mathrm{pm})$ & 0 & 17.1 & 4.3 & 10.2 \\
$\sigma_{\delta}(\times 0.001)$ & 0.77 & 1.05 & 1.05 & 1.10 \\
damping time $\tau_{x}(\mathrm{~ms})$ & 47 & 21 & 22 & 17 \\
damping time $\tau_{y}(\mathrm{~ms})$ & 112 & 29 & 29 & 21 \\
damping time $\tau_{s}(\mathrm{~ms})$ & 176 & 17 & 18 & 12 \\
\hline
\end{tabular}


IBS effects may differ for the two approaches of generating vertical emittance, i.e., with full coupling or with vertical damping wiggler, we did IBS calculation for both cases for the PEP-X compatible lattice with the high energy approximation model $[3,9]$. Similar to the nominal PEP-X, we assume the Coulomb $\log$ function is $(\log )=11$. For the full coupling case, a horizontal damping wiggler is put into the model to reduce emittance. The wiggler parameters are the same as the vertical damping wiggler. For the case corresponding to VDW-3 in Table 1 (i.e., with peak field $1.5 \mathrm{~T}$, wiggler period $100 \mathrm{~mm}$ and wiggler length $89.4 \mathrm{~m}$ ), the emittances are $10.5 \mathrm{pm}$ for both planes. For the vertical wiggler case, a small linear coupling ratio of $\epsilon_{y} / \epsilon_{x}=0.001$ is assumed. The vertical emittance is almost entirely generated with the vertical damping wiggler. In existing third generation light sources, linear coupling and vertical dispersion correction can routinely keep the emittance ratio at the $0.1 \%$ level or below.

The emittances vs. beam current for the two cases with IBS effects are shown in Figure 6. The bunch length is assumed to be $\sigma_{z}=2.7 \mathrm{~mm}$ at the zero current limit, corresponding to an RF gap voltage of $6 \mathrm{MV}$ with the $476.0 \mathrm{MHz}$ rf system. The ratio of bunch length over momentum spread is kept constant in the IBS calculation. The total number of bunches is assumed to be 3300 . For the vertical DW case, the horizontal emittance has a significant increase. But the vertical emittance growth is very small. This is because the vertical dispersion is confined to inside the damping wiggler, which constitutes only a small fraction of the ring, while the horizontal dispersion is present at all arc areas. In addition, the vertical dispersion is much smaller than the horizontal dispersion while the horizontal and vertical emittances at zero current are nearly the same. This is because the average bending field in the damping wiggler is much stronger than in the bending magnets. Overall the vertical IBS growth rate is much smaller than the horizontal plane because the IBS growth rate is proportional to the dispersion invariant averaged over the ring circumference.

The distribution of the IBS growth rate for the vertical DW case is shown in Figure 7 for the case with a $200 \mathrm{~mA}$ total current. For this case, the average IBS growth rates for $x, y, p$ directions are $26.1 \mathrm{~s}^{-1}, 0.20 \mathrm{~s}^{-1}$ and $8.4 \mathrm{~s}^{-1}$, 




Figure 6: Emittance growth vs total beam current, assuming a uniform current distribution in 3300 bunches and a zero-current bunch length of $2.7 \mathrm{~mm}$.

respectively. The corresponding emittances are $\epsilon_{x}=22.3 \mathrm{pm}$ and $\epsilon_{y}=10.3 \mathrm{pm}$ and the momentum spread is $\sigma_{\delta}=1.16 \times 10^{-3}$. If harmonic cavities are used to lengthen the bunch to $\sigma_{z}=5 \mathrm{~mm}$, the $x, y, p$ IBS growth rates become $18.8 \mathrm{~s}^{-1}$, $0.13 \mathrm{~s}^{-1}$ and $5.5 \mathrm{~s}^{-1}$, respectively, and the emittances and the momentum spread become $\epsilon_{x}=18.4 \mathrm{pm}, \epsilon_{y}=10.25 \mathrm{pm}$ and $\sigma_{\delta}=1.13 \times 10^{-3}$.

\section{Conclusion}

For ultimate storage rings that plan for off-axis injection, we propose the use of vertical damping wiggler (with horizontal magnetic field) to generate vertical emittance in order to obtain round beams. This approach has an advantage over the approach of generating round beams with $100 \%$ coupling because it does not couple the large amplitude horizontal oscillation of the injected beam to the vertical plane and therefore the small vertical apertures in the ring does not pose severe limitation to the dynamic aperture. It is shown that for damping wigglers with reasonably small wiggler period (e.g., $\lambda_{w}=100 \mathrm{~mm}$ ), the total emittances of the two approaches are nearly equal. The vertical damping wigglers causes an increase to the momentum spread. The amount of momentum spread increase would be the same if horizontal damping wigglers of identical parameters are used. 


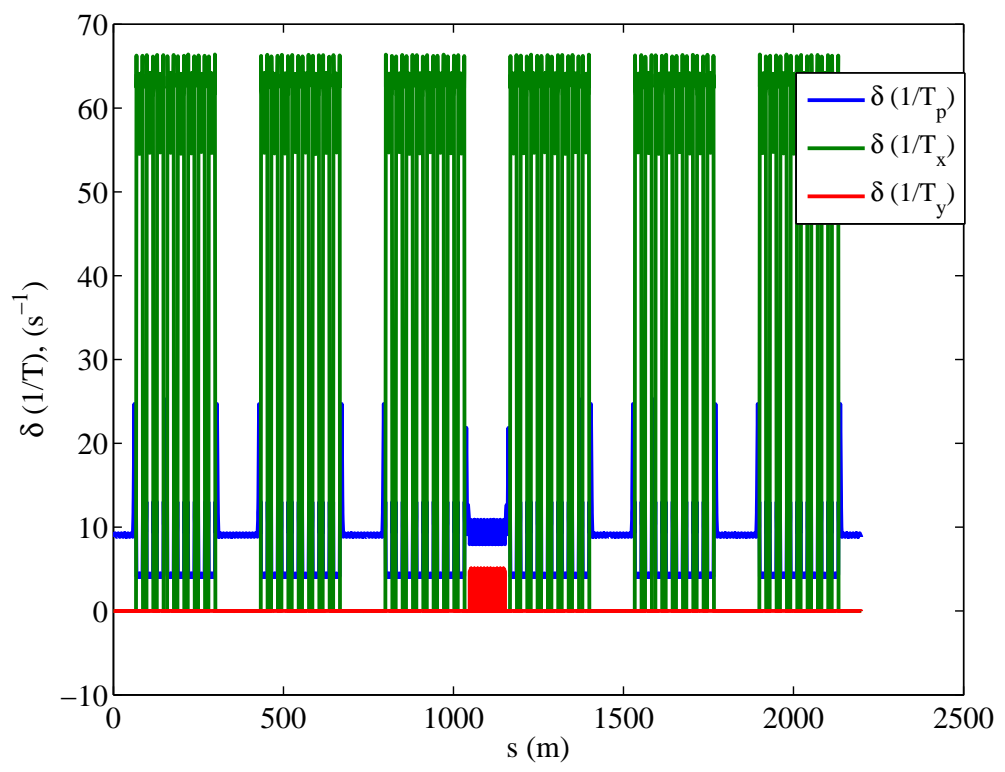

Figure 7: The distribution of local IBS growth rate for the three dimensions for a beam current of $200 \mathrm{~mA}$ in 3300 bunches.

A PEP-X compatible lattice is designed to demonstrate this approach. The bare lattice horizontal emittance is $36.5 \mathrm{pm}$ at $4.5 \mathrm{GeV}$. When a $90-\mathrm{m}$ long vertical damping wiggler with peak field at $1.5 \mathrm{~T}$ and wiggler period at 100 $\mathrm{mm}$ is put into one of the long straight sections, the horizontal and vertical emittances are $13 \mathrm{pm}$ and $10 \mathrm{pm}$, respectively. The rms momentum spread is $1.1 \times 10^{-3}$. Intrabeam scattering is calculated for this case. For $200 \mathrm{~mA}$ beam current in 3300 bunches and a zero-current bunch length of $\sigma_{z}=2.7 \mathrm{~mm}$, the horizontal and vertical emittance become $22.3 \mathrm{pm}$ and $10.3 \mathrm{pm}$, respectively. The rms momentum spread is $1.16 \times 10^{-3}$.

\section{Acknowledgment}

The study is supported by DOE Contract No. DE-AC02-76SF00515.

[1] X. Huang, "A PEP-X lattice with vertical emittance by damping wigglers", SSRL-AP-note 51, July 2013. 
[2] A. Bogomyagkov, et al, presentation at the 4th Low Emittance Rings Workshop, September 2014.

[3] Y. Cai, et al, Phys. Rev. ST Accel. Beams, 054002 (2012).

[4] S. Y. Lee, Accelerator Physics, World Scientific (1999).

[5] A. Chao, M. Tigner, ed., Handbook of accelerator physics and engineering, p. 185-186, World Scientific (1998).

[6] A $7.5 \mathrm{~cm}$ minimum magnet separation is reserved in MAX-IV design. See Detailed Design Report on the MAX IV Facility, https://www.maxlab.lu.se/node/1136, August 2010, chapter 2.

[7] T. Rabedeau, private communications (2013).

[8] H. Grote, F.C. Iselin, The MAD Program User's Manual, CERN/SL/90-13 (1990).

[9] K. Bane, EPAC 2002, Paris, France (2002) 